\title{
Battle between Human and Viruses: An Antidisease Visual Approach on Viruses' Aduptive Nature to Concentrate as an Endurance Endeavors
}

\author{
${ }^{1}$ Dr. Rina Girish Mehta ( B.D.S.,M.D.S.,Oral Pathology) \\ Lecturer, FDS, DDU, \\ Nadiad, Gujarat, India \\ ${ }^{2}$ Rami Kamlesh (USA)
}

\begin{abstract}
Humans were battling for survival before evolution till our modern lifestyle. Amongst all, viruses are such tiny organisms keep infecting a humans a decades ago and spreading in universe by many routes/pathways. Animal to human transmission is triggered outbreaks claiming thousands of lives, in a period of time by different species of viruses. RNA genome viruses are assumed to more virulent than DNA genome one due to its complex structural instability, environmental adoptability, mutational properties. Study of its entry in human body, attachment and replication into host cells, response of host defence cells by innate and adaptive immunity is helpful to know virus' mild to lethal pathogenic nature. Marburg, Ebola, HIV,influenza, SARS-COV, and 2019 -nCoV( COVID 19 /SARS-COV2) are few deadly threats of a battle. Study by lab investigation, vaccines, antiviral drugs need to focus on antidisease approach then anti pathology visionary to eradicate and protect human from viruses' bioweapon use.
\end{abstract}

Keywords:- Viruses, immunity, 2019-n COVID, antidisease approach.

\section{INRRODUCTION}

Virus is a teentsy pathogen, exists as an independent particle found in every echo system .Its structural genetic material is a molecules of either DNA or RNA, encodes 4 proteins in a simple to $100-200$ proteins in a complex form.

Virion, an entire virus particle contains nucleic acid may be single or double stranded enclosed within protein coat/ capsid .Though less DNA and RNA material with limited number of genes, virus encodes all genetic information to form large capsid through this nucleic acid ,so named nucleocapsid . Outer core is called lipid envelop.

Shapes of viruses vary from simple helicle (TMVtobacco mosaic virus), icosahedral form to more complex structures. It is a contagious, replicate solely inside the living cells of the either animals and plants and disperse sequence of genomes by multiplication and mutation .
Marburg, Ebola, Rabies , HIV,Herpes simplex, Smallpox, Hanta, influenza, Dengue, Rota, SARS-CoV -2( 2019-nCoV) are few deadliest viruses had put nature and lives in danger through endemic, epidemic or pandemic spread. Pathogenicity and existance of each one are depend on their diverse aduptive ecology/nature with different environs. Cure of infection, possible immunization at community level and / or global eradication are a challenge for medical profession which necessitate to explore virus with time.

\section{DISCUSSION}

\section{> MARBURG VIRUS:}

Marburg virus is a filamentous negative sense, non segmented RNA virus from filoviridae family which encodes seven genes. ${ }^{1}$ It was first found in 1967, germany, continued outbreak till 2017 uganda, with $4^{\text {th }}$ number of infection with upto $90 \%$ fatality. ${ }^{2}$ Virus infected bats infects humans through abraded skin and mucosa, low aerosol transmission and fatal parenteral exposure. ${ }^{3}$ Incubation period 2-21days. Accurate disease progression begins with flu-like symptoms chills, sore throat, headache, painful muscles and joints, alveolar congestion. Other symptoms include bleeding from mucosa, fever, bloody vomitus and watery diarrhea. ${ }^{3}$ Diffuse skin rashes with petechiae initially and aggressiveness, seizures and coma in later stage. Mild to severe necrosis in spleen , liver ,kidney and lymphoid tissue depletion by apoptosis without viral antigen ${ }^{2}$ Death due to irregular immune response , dehydration and haemorrhage with intravascular coagulation and survival sequels visible with arthitis, conjunctivitis, pschycological upset. Social separation is helpful for recovery. ${ }^{3}$ It was programmed as biological weapon during cold war for offense and defence.

\section{$>$ EBOLA VIRUS:}

Ebola virus is same as Marburg virus in structure, negative-sense, single-stranded RNA viruses of ebolavirus genome and Filoviridae family, with highly infectious nature. It was first recognized near the Ebola River valley during in Zaire in 1976with current outbreak in 2018-19 with epidemiological data of 40 years 4,5 Human transmission of Infection occurs through close contact with infected animals' body fluids. ${ }^{5}$ It also shows $50-90 \%$ lethality due to hemorrhagic symptoms by endothelial cell toxicity and hypovolemic shocks. ${ }^{4,5}$ Infection course is 14- 
21days. ${ }^{4}$ Virus matrix protein provides important insights for its replication and rapid progressive pathogenesis. Knowledge of innate immunity requires study about production secreted glycoprotein antibody after infection (sGP) which is not produced by MARV. Infection is visualized in early and preferred targated cells, Monocytes (dendritic cells (DCs)) and macrophages by ebola virus. ${ }^{5,6}$ Virus evades immune responses like that in HIV5 and herpes virus ${ }^{4}$

\section{> RABIES VIRUS: (RABV)}

Rabies virus is rabere (to be made in latin), bullet shaped enveloped virion, negative sense single stranded RNA genome 7, It is a zoonotic virus that of genus Lyssavirus of Rhabdoviridae family and of neuroinvasive nature. It was first found in Babylon monkey in the 2300 BC in Egypt .Louis Pasteur had identified in 1880 with first vaccine immunization applied in $1885^{\prime}$. It is fatal, progressive, $7^{\text {th }}$ grade endemic infection of nervous tissue affecting all warm-blooded animals, with panic health issue in asians and Africans. Amongst multiple genotypes most are causing human infection. Transmission through rabid animal bite at peripheral site and gained entry of infected saliva through open, abraded skin wounds, Raba particle inhalation, organ and cornea transplants. Bites with bleeding are of acute onset and most risky. ${ }^{\mathbf{1 0}}$ More than 60000 deaths per year are counted worldwide. RABV eclipse phase is 2 weeks to 6 years. ${ }^{10}$ Retrograde axonal centrifugal spread occurs along with PNS path in ascending manner from CNS after invasion of CNS from peripheral site of virus uptake into body. ${ }^{8} 10$ days after virus gain entry into human body, itching, pain and paresthesia are present at the bite wound site. Neuronal dysfunction, ${ }^{9}$ cell to cell spread, ${ }^{8}$ N2o induced toxicitity are present. Negri bodies, neurovirulence ${ }^{8}$ but no neuron apoptosis and inversely related pathogenicity to virus replication are definite characteristics of RV. Symptoms include degeneration of ganglion cells, salivary, adrenal and lacrimal glands, thrombosis and haemorrhages in vascular system and, generalised flaccid paralysis. Preventive and post exposure immunization by injectable live or dead viruses with protocol of 0,7,28 days and booster dose every year is recommended for control of rabies endemically.

\section{> HIV VIRUS:}

HIV virus is a retro virus of Lentivirus ${ }^{13}$ family with long letancy ${ }^{13}$ persistent replication and CNS involvement. ${ }^{11} \mathrm{HIV}-1$ And HIV -2 are the viral antigen types . RNA genome Of HIV-1 is 2 single stranded molecules enclosed in protein core and lipid membrane It cannot survive outside the bloodstream or lymphatic tissue and easily inactivated by the exposure to common detergents and disinfectants. ${ }^{13}$. Virus was first introduced in humans through African chimpanzees in1920-1940. Virus enters into body through mucous membranes, injured skin or mucosa and by parenteral path. HIV can be transmitted through blood,plasma or serum organ transplantion and artificial insemination. All CD4-positive cells 13(permanent reservoir of virus )such as, dendritic cells ,T helper cells, macrophages ${ }^{13}$ and astrocytes are susceptible to HIV. Assumption of incubation period is several weeks at $4 *$ c. Sexually transmitted virus attaches first to dendritic cells (e.g. Langerhans cells) or macrophages ${ }^{\mathbf{1 2}}$ then reach to Blood monocytes, where it replicates. From day one to 14 days period, HIV is detected in lymphoid tissue, lymphnodes, whole body with nervous system involvement .HIV 2 pathogenicity is lower than HIV 1 . HIV -1 infection course is pandemic. ${ }^{11}$ Frequent period of illness increases with its course of infection includes mild fever, diarrhoea, fatigue, weight loss, opportunistic infections ${ }^{13}$, neoplasms ${ }^{13}$ , impaired neuronal and cerebral functions, oral and skin infections highlighten generalized immunodeficiency. ${ }^{13}$ ELISA and Western blot test, NAT assay provides confirmatory investigation. Infection course is chronic and fatal as trial of vaccine since 1983 is unsuccessful so no eradication virus is possible till date. ${ }^{\mathbf{1 1}}$ Reduction of HIV infection through blood and plasma donor is possible after 2004 with safe and quarantine collections.

\section{$>$ HERPES SIMPLEX VIRUS (HSV):}

HSV is a neurotropic double-stranded DNA virus which belongs to Alphaherpesvirinae ( Herpesviridae) family ${ }^{15}$. Herpes means to creep/crawl ${ }^{17}$ as cold sore, described in Rome (AD100) as blister on ladies' lips. ${ }^{18}$ Herpes was found near 1940s . Orolabial infection in the late $19^{\text {th }}$ century with 2 types identification near 1960 's. Amongst two types ${ }^{14,18}$ HSV1 is more common causes mouth, throat, face, eye, and CNS infections, whereas HSV $-2^{15}$ causes primarily anogenital infections. ${ }^{17}$ Both are unique biologically in neurovirulance, latency and reactivation of infection. Close contacts at virus shedding ${ }^{14}$ secretory surface of infected one plays a role for virus transmission eg. Perinatal infection at the time of child birth. ${ }^{14}$ Virus is inactivated at room temperature and by drying, so no aerosol or fomite spread of virus is 17,18 Virus enters through abraded skin or mucous membrane ${ }^{14}$ replicates in the ganglion cells. Primary infection starts within five days of short incubation period, inoculate in ganglions. Centrifugal migration spread of virions to mucosal skins through trigeminal ${ }^{18}$ and other ganglions by sensory and motor nerve infection. Initial crop of vesicles visualized with the large surface area with recovery from neural tissue. Due to contagious nature, virus spreads via autoinoculation at distance from neurons innervating via inoculation. virus allows disease extension. Ganglion are the reservoirs of viruses due to lifelong latency of virus $^{14,15,16} \mathrm{HSV}$ infection causes orofacial and genital herpes ${ }^{14}$, herpetic whitlow, herpes keratitis and encephalitis ${ }^{14}$, knife cut erosion in skin, herpetic sycosis is infection hair follicles, eczema herpeticum, multiple, round, superficial oral ulcers ${ }^{18}$ accompanied by acute gingivitis. Neonatal herpes is morbid and fatal. Recurrent outbreaks are common amongst the immunosuppressed persons. Prevention by use of condomand antiviral drugs. 281

\section{SMALLPOX (Variola):}

Human existence of variola virus since1763,orthopox genus $^{20,21}$ contagious $^{19}$, double stranded DNA virus ${ }^{19,22}$ structured with core ,lateral body, membrane and envelop. Virus spreads in human to human contacts ${ }^{19}$ by air born 
droplets. Variola major ${ }^{20}$ affects $90 \%$ and variola minor affects only $2 \%^{19}$ in previously vaccinated ones.. Smallpox virus is resistant to environmental changes. ${ }^{21}$ Its a infection of $\operatorname{skin}^{20}$ (squamous epithelium )and mucous membrane\%, oropharynx. ${ }^{20}$ It is completely eradicated by 1980 (WHO) with vaccines. Belief of use of massive endemic virus as bioterrorist weapons ${ }^{19,20}$ was programmed for invading ohio river velly by the English ${ }^{19}$. Pathogenicity visualized after direct contact, body fluid and aerosol inhalation from infected person. Symptoms appear within 7-17 days after exposure with high fever, spike for 3-5days, myalgia, and headache. A maculopapular rashes ${ }^{22}$ erupt on the face and neck, convert as papules become round and firm vesicle within dermis and measure $2-5 \mathrm{~mm}$ in diameter.Lethal variant ${ }^{22}$ flat small pox' in children was without pustule, vesicle and skin eruption, ${ }^{20}$ After 9-10 days of exposure crusting and scabs ${ }^{20,22}$ will appear . Pitting scars ${ }^{21,22}$ appear after infection resolution.. Death may occur due to toxaemia , pneumonia. ${ }^{19}$ Morbidity as pockmarks ${ }^{19,20,22}$ are found in upto $80 \%$ and blindness due to viral keratitis ${ }^{19}$ upto $1 \%$ of in virally infected survivors. Arthritis, encephalitis resulting in either retardation or death of a person is rare. Patients' isolation and moniterization for fever , swab sample reports, PCR assay\%, health supportive measures and successful ring vaccination chain has proven helpful for virus eradication from the world in1977. ${ }^{19}$

\section{> HANTA VIRUS:}

Hanta virus is round, pleomorphic or tubular particle of Hantavirus genus within the family of Bunyaviridae of three-part segmented negative-sense, single stranded RNA genomes*. Disease named as Virus-induced haemorrhagic fever with renal syndrome (HFRS) are found in Russia as early as 1913; Human/ Huntavirus pulmonary syndrome (HPS) in 1993 named by Hughes et al., 1993and as hantavirus cardiopulmonary syndrome (HCPS) (Hallin et al., 1996) as of today. ${ }^{23,24}$ Hantaan virus (HTNV), was identified in 1978 by Lee et al. near small river called Hantaan. Until now, 21 species and more than 30 genotypes can be found in the world ${ }^{23}$ Virus is transmitted in humans by excreta of rodents and insectivores, arthropods when becomes airborne, rodent bites and blood transfusion.. Humans are dead end host for this virus. ${ }^{24}$ Entry into cell facilitated only by fusion with endosomes. ${ }^{25}$ Global estimation by Jonsson et al in 2010,that aproximately1,50000 people get infected per year ${ }^{23,25}$ by fatal hemorrhagic fever with renal syndrome (HFRS), clinical symptoms include fever, renal dysfunction, haemorrhage / leakage due to increase microvascular permeability and shock. Hantavirus cardiopulmonary syndrome (HCPS) is with added symptoms of gastrointestinal tract followed by non-cardiogenic pulmonary oedema, leads to shock ${ }^{26}$ The clinical course is subdivided into five phases: Febrile, hypotensive, oliguric, diuretic, and convalescent. Phoptophobia, conjuctival bleeding, petechiae, hematuria , proteinuria, hypotension and shock, and at last upto renal failure within $3 \mathrm{wk}$ to 6 month .vaccination is not successful .innate immunity is developed after infection. No antiviral drug can be effective, only give supportive therapeutic measures.

\section{$>$ INFLUENZA VIRUS :}

Influenza virus, human respitatory virus, possess RNA genome of Orthomyxoviridae family. Influenza A viruses (IAV), influenza B (periodically epidemic) viruses are virulent to humans and influenza $\mathrm{C}$ (endemic and mild) . Virus causes seasonal with annual winter outbreaks, periodic, endemic, unpredictable pandemic infections. Symptoms include high fever, body aches, and fatigue. Coryza, cough, headache, prostration, malaise, and inflammation of the upper respiratory tree and trachea persist for 7 to 10 days, feeling Weakness and fatigue present for days to weeks).People of all ages are affected but school-age children and infants aged and physically unfits have disease severity. Croup /laryngotracheitis can be a serious complication in small children. From influenza A viruses, hemorrhagic bronchitis, pneumonia are sever complications in patients suffering from pulmonary or cardiac disease, or diabetes mellitus. Four pandemics of 20th century: Spanish influenza (H1N1) in 1918/1919(most devastating bacterial pneumonia), Asian influenza (H2N2) in 1957, Hong Kong influenza (H3N2) in 1968, and H1N1 influenza in $2009^{28}$ worst influenza virus pandemic senario in 1918, killed approximately 50 million people worldwide ${ }^{27,29}$ Influenza A H1N1is pandemic in $2009^{27}$ and avian influenza (HPAI) virus of $\mathrm{H} 5 \mathrm{~N} 1$ causes highly pathogenic pandemic emergency ${ }^{29}$ Reverse genetics of virus indicates its cloning ${ }^{27}$ in 1999 . IAV infects Alveolar macrophages that migrate to influenza-infected lungs play a pathogenic role in pulmonary inflammation. ${ }^{27}$ Innate immunity have two distinct role while aduptive immunity (antigen specific antibody by $\mathrm{T}$ and $\mathrm{B}$ cells) recovers the host from infection and protect the host from reinfection.

\section{$>$ DENGUE VIRUS:}

Dengue is an acute infectious, endemic ${ }^{31,33}(128$ countries with indian subcontinents is epicenter) viral illness with self limiting fever(5-7days) to life threatening Dengue hemorrhagic fever(DHF) . Chinese medical encyclopedia from Jin Dynasty (265-420AD) which referred to a "water poison" by flying insects. Break Bone Fever was named by Benjamin Rush in 1789 (Philadelphia epidemic)named "bilious remitting fever". The term dengue fever came into use after $1828 .{ }^{34}$ It is caused by RNA virus of family, has 4 serotypes: DENVs $1-4^{33}$. , spread by female Aedes Albopictus (DHF) ${ }^{30,31,32}$ and Aedes Aegypty mosquitoes ${ }^{33}$ bites. The life cycle of Aedes mosquito depending upon the extent of feeding lasts for 8 10 days at room temperature. It consists of two phases: aquatic (larvae, pupae) and terrestrial (eggs, adults) phase $^{31}$.In humans, the mosquito delivers virus in skin epithelium, infects and replicates in the cells of mononuclear lineage (monocytes, dendritic cells, macrophages, and Langerhans cells). These cells carry the virus to lymph nodes, where it replicates, viremia occurs. It is followed by systemic infection of liver, lungs, and spleen. Symptoms similar as typhoid or Typhoid Fever (TF) characterized by fever with body ache, nausea, vomiting, red skin spots ${ }^{32}$, petechiae on extremities, respiratory symptoms, decreased apatite, nosebleeds! later DHF and dengue shock syndrome (DSS)in children, adolescents and adults ${ }^{32}$. Dengue fever still occur in 
Indonatia every year ${ }^{30}$. Number of sufferers from 20142019 can involve heart, kidneys, brain, and lungs along with above mild symptoms to unstable breathing, fainting and death due to shock .DHF with critical plasma loss may be life threatening if not treated properly, characterized by a rapid, weak pulse with narrowing pulse pressure $(<20 \mathrm{mmofHg})$, cold clammy skin, and restlessness. The patient may die within $12-24 \mathrm{~h}$ of going into shock or recover rapidly with volume replacement therapy ${ }^{31}$. Life long immunity with any of serotypes after primary infection does not provide protection against other serotypes for secondary infection. ${ }^{31}$ Till date no drug is available for dengue. Bed rest, oral rehydration, and paracetamol as an antipyretic and analgesic, Patient's health monitoring through blood tests from fever day 3 onwards,patient recovers within $12-48 \mathrm{~h}$ of fluid therapy are performed till the condition improves Licensed Sanofi Pasteur dengue vaccine Dengvaxia is under trial of its success. 31

\section{$>$ ROTA VIRUS :}

It is Rotavirus genus of the Reoviridae of a Reoviridae Family, genome, composed of 11 segmented double stranded RNA. Its virion is complex, non enveloped and surrounded triple layered capsid. ${ }^{35}$ It has six structural and six nonstructural protein. From A to E genus , A to $\mathrm{C}$ Infects humans and all infects animals. Age dependant immunity, so it is restricted upto children of 5 years/youngs. ${ }^{36}$ Rotaviruses replicate in the nondividing mature enterocytes near the tips of the villi. Infection alters small intestinal epithelial motility function results in malabsorptive diarrhea and inflammation. Enterocyte vacuolization and loss or villus blunting due to ischemia and crypt hyperplasia, result in the transit of osmotically active undigested bolus of mono and disaccharides, carbohydrates, fats, and proteins into the colon. The colon is unable to absorb sufficient water, leading to an osmotic diarrhoea with viruses in stool10 10 particles /gram. Malnutrition increases the severity of rotaviruses infection. Approved vaccines was recommended in an early 80's to $3^{\text {rd }}$ Generation RotaTeq and Rotarix vaccines ${ }^{37}$ but later withdrawn due to its adverse effects. Pathogenesis of Intestinal and Systemic rotavirus Infection depends on person's health, virus replications, reinfection, herd or individual immunity after primary infection.

\section{SARS-COV:}

Severe acute respiratory syndrome (SARS) is an infectious viral disease of the lower respiratory tract. Corona viruses are enveloped, single-stranded of largest genome $^{40}$, positive-sense RNA viruses of coronaviridae ${ }^{38}$ family. Virus replicates in upper respiratory tract. SARS coronavirus causes collapse of tracheobronchial alveolar epithelial cell by diffuse edema, desquamation ${ }^{38}$, fibrosis, atrophy, thrombosis, necrosis ${ }^{38}$, degeneration and apoptosis like deadly effects. Damage of several organs like brain, intestine, glands, spleen, lymphnodes, kidneys and immune cells injury was seems by all above deadly effects. No definite effects on bone marrow. The pathogenesis of SARS is highly complex seems that both abnormal immune responses and injury to immune cells with lack of
ACE2 expression may have a key role . Multinucleated syncytial cells ${ }^{38}$ are common histological structure found in many viral infection with no report of presence in $\mathrm{H} 5 \mathrm{~N} 1$ type infection ${ }^{38}$.Viral antigen, nucleic acid and virions are found on the bronchiolar epithelial cells ${ }^{38}$. SARS was first emerged in China's Guangdong Province in November $2002^{38}$, pandemic to epidemic between 2003 and sporadic till spring 2004. Bat species is the natural reservoir of virus. virus transmission to and among humans is by direct contact, droplet, and airborne and is isolated from cat ,dog like animal. Virus is isolated from faecal and urinary samples. Flu-like symptoms including fever, chills, cough, and malaise, with the $70 \%$ of the patients, suffer from shortness of breath and recurrent or persistent fever, whereas the $30 \%$ show improved after first week, 20 to $30 \%$ of patients were required intensive care treatment with mechanical ventilation in 2003. Thrombocytopenia and lymphopenia ${ }^{38}$ have been frequently detected in SARS patients and $6.8 \%$ fatality rate in younger than $43 \%$ in 60 years of age were the estimated autopsied reports since the first outbreak in 2003.

\section{$>$ COVID -19 (2019-nCOV)}

COVID-19 is a new viral infection caused by the severe acute respiratory coronavirus 2 (SARS-CoV2).SARS-CoV-2, a new coronavirus( coronam $=$ crown $)^{41}$ found in WUHAN in december $2019^{42}$ is of family Coronaviridae, subfamily Coronavirinae, which have solar corona[ virion with $9-12 \mathrm{~nm}$ long spikes. $^{42}$ Virus is spherical $7^{\text {th }}$, non segmented, largest $(18 \mathrm{~kb}$ in length $\{27$ to $32 \mathrm{~kb}\}$ ), spherical, enveloped, highly transmissible, ${ }^{43}$ contagious, positive-stranded RNA virus ${ }^{41,43}$ Amongst alpha, beta, gamma, and delta categaries, SARScov2 is of Beta family ${ }^{41,42,43}$. Encoding genome have multiple structural proteins for infection in host by fusion, assembly and formation of virus and non structural proteins for replication and transcription of virus . Envelop , membrane and spike proteins acquire its peculiar pathogenic properties ${ }^{44}$ SARS(Severe acute respiratory syndrome)-CoV in $2003^{43}$, MERS (middle east respiratory syndrome)-CoV emerged in 2012, ${ }^{43}$ and SARS-CoV-2 (WHO named COVID-19)deadly affect multiple organs along with severe respiratory illness. Pangolin and Bat are the reservoir of virus.

Out of six Human Coronavirus species ( $\mathrm{HCoV})$, four of them are a causative virus for common cold symptoms in immunocompromised individuals. Anlytical genomes lineage $\mathrm{A}$ and $\mathrm{C}$ types were found in American and Europeans, while the B types was prevalent in East Asia might needs mutation for outer country spread. The lineage $\mathrm{C}$ was prevalent in France, Italy, Sweden, England, California, Brazil, Singapore, Hong Kong ${ }^{43}$ Fever, cough ,fatigue and death are common symptoms of SARS coV2. ${ }^{42}$ More complex and large clove shaped spikes proteins ${ }^{43}$ [with internal fusion peptide, double cleavage sites, and long six-helix bundle [permit larger binding affinity of virus with host receptor ACE2(Angiotensin -converting enzyme 2)might explain faster spread of SARS-CoV-2 than that of SARS-CoV. ${ }^{42}$ Above 20million confirmed cases with more than 7lakhs deaths(till date 
written),brought major health emergency threats, social issues as well as drastic economical crisis, as of worldwide outbreak a pandemic. ${ }^{43}$

\section{CONCLUSION}

Any viral nucleic acid detected by PCR is actually infectious virus. Positive sense virus is similar to mRNA, immediately translated by host cells whereas negative sense virus is complemantary to mRNA so before translation it must need to convert in positive sense .To degrade and recycle easily when genes are activated, RNA Has to transcribe and translate, RNA is single stranded for unstable nature. RNA virus have high mutation rates that allow fast evolution. RNA viruses have high rates of sequence divergence, small genomes recombination, and reverse transcription machinery. poilo virus was first man made virus generated without natural templet were of Polio virus and $\mathrm{Q}><174$ bacteriophage. ${ }^{40}$ Virus isolation by culture, histopathology, seroanalysis, molecular techniques of polymerase chain reaction (PCR), ReversetranscriptionPCR and real-time PCR ${ }^{39,40}$ are in use today. Stopping viral replication by target stopping of genetic process of cleaving/splitting the DNA/RNA strands will able to stop their correct functioning.

With a period of time, repeated infections in humans from bats, a reservoir of virus of coronaviridae family with structural changes showed different virulent effects. Infectious disease study review may guide and provide lessons to FOCUS more on to shift on anti disease approach adopted by bats rather than traditional anti pathogen approach to control infectious disease in humans.

\section{REFERENCES}

[1]. Adrianna P. P. Zhang,a Zachary A. Bornholdt,a Dafna M. Abelson,a Erica Ollmann Saphirea, Crystal Structure of Marburg Virus VP24 May 2014 Volume 88 Number 10 Journal of Virology p. 5859-5863

[2]. Shifflett and Marzi Marburg virus pathogenesis differences and similarities in humans and animal models Virology Journal (2019) 16:165).

[3]. Masfique Mehedi1,2,*, Allison Groseth2,*, Heinz Feldmann1,2, and Hideki Ebihara $\dagger$, Clinical aspects of Marburg hemorrhagic fever Future Virol. 2011 September ; 6(9): 1091-1106

[4]. MINIREVIEW Nancy Sullivan, Zhi-Yong Yang, and Gary J. Nabel* Ebola Virus Pathogenesis: Implications for Vaccines and Therapies JOURNAL OF VIROLOGY, Sept. 2003, p. 9733-9737 Vol. 77, No. 18

[5]. César Muñoz-Fontela Anita K. McElroy Ebola Virus Disease in Humans: Pathophysiology and Immunity Marburg- and Ebolaviruses Current Topics in Microbiology and Immunology . 27 June 2017 pp 141-169

[6]. Jeffrey E Lee Erica Ollmann Saphire Ebolavirus glycoprotein structure and mechanism of entry Future Virol. 2009 ; 4(6): 621-635.
[7]. Joshua A. Horwitza,b, Simon Jennia, Stephen C. Harrisona,c, $1 \square$, and Sean P. J. Whelanb Structure of a rabies virus polymerase complex from electron cryomicroscopy PNAS | January 28, 2020 | vol. 117 | no. 4 | 2099-210,

[8]. Bernhard Dietzschold. Concepts in the pathogenesis of rabies Future Virol. 2008 September ; 3(5): 481490

[9]. Alan C Jackson Rabies pathogenesis Journal of NeuroVirology, 8: 267- 269, $6^{\text {th }}$ may 2002

[10]. Rajendra Singh, Karam Pal Singh, Susan Cherian, Mani Saminathan, Sanjay Kapoor, G.B. Manjunatha Reddy, Shibani Panda \& Kuldeep Dhama Rabies epidemiology, pathogens is, public health concerns and advances indiagnosis and control: a comprehensive review, Veterinary Quarterly, 37:1, 212-251June 2017

[11]. Andrea Rubbert, Georg Behrens and Mario Ostrowski Archieves: structre of HIV-1 Pathogenesis of HIV-1 Infection 28 october2011;13:26

[12]. German Advisory Committee Blood (Arbeitskreis Blut), Subgroup 'Assessment of Pathogens Human Immunodeficiency Virus (HIV) Transfus Med Hemother 2016;43:203-222.

[13]. Emanuele Fanales-Belasio(a), Mariangela Raimondo(b), Barbara Suligoi(b) and Stefano Buttò(a) HIV virology and pathogenetic mechanisms of infection: a brief overview (a)Ann Ist Super Sanità 2010 | Vol. 46, No. 1: 5-14

[14]. Herpes Simplex Virus - Genital National STD Curriculum PDF created August 8, 2020

[15]. Review Article Shahid K. Sukhbir Neonatal Herpes Simplex Infection Hindawi Publishing Corporation ISRN Infectious Diseases Volume 2013, Article ID 473053, 7 pages

[16]. Tracy J. Smith,1 Lynda A. Morrison,2 and David A. Leib1,3* Pathogenesis of Herpes Simplex Virus Type 2 Virion Host Shutoff (vhs) Mutants JOURNAL OF VIROLOGY, Mar. 2002, p. 2054-2061 Vol. 76, No. 5

[17]. Author : Folusakin O Ayoade, MD: Chief editor:Michael stuart Bronze infectious disease Herpes Simplex Medscape Drug and disease> updated Mar 01, 2018

[18]. Murtaza Mustafa 1, EM.Illzam 2, RK.Muniandy 3, AM.Sharifah 4, MK.Nang 5, B.Ramesh 6 Herpes simplex virus infections, Pathophysiology and Management IOSR Journal of Dental and Medical Sciences (IOSR-JDMS) Volume 15, Issue 2 Ver. IV (Feb. 2016) PP 72-78

[19]. Roy Guharoy, Robert Panzik, John A Noviasky, Edward P Krenzelok, and Donald C Blair Infectious Diseases Smallpox: Clinical Features, Prevention, and Management The Annals of Pharmacotherapy 2004april;38:440-7

[20]. Mike Bray 1 and Mark Buller 2 CONFRONTING BIOLOGICAL WEAPONS CID Looking Back at Smallpox Clinical Infectious Diseases :15 march2004; 38:882-9. 
[21]. Theves et al. , P. Biagini2 and E. Crubezy1 C. Th_eves1, P. Biagini2 and E. Crub_ezy1 The rediscovery of smallpox Clin Microbiol Infect 2014; 20: $210-218$

[22]. MARK L.BULLER* AND GREGORY J.Poxvirus Pathogenesis R PALUMBOMICROBIOLOGICAL REVIEWS.Mar.1991,p.80-122 Vol.55,No.1

[23]. Walter Muranyi,* Udo Bahr,* Martin Zeier,* and Fokko J. van der Woude† Hantavirus Infection Am Soc Nephrol 16: 3669-3679, 2005

[24]. Jussi Hepojoki, Tomas Strandin, Hilkka Lankinen and Antti Vaheri Hantavirus structure - molecular interactions behind the scene Journal of General Virology (2012), 93, 1631-1644

[25]. J. Battisti,1 Yong-Kyu Chu, $\uparrow \uparrow$ Paul R. Chipman,1 Bärbel Kaufmann,1 Colleen B. Jonsson, $2 \ddagger$ and Michael G. Rossmann1 Structural Studies of Hantaan Virus_Anthony JOURNAL OF VIROLOGY, Jan. 2011, p. 835-841 Vol. 85, No. 2

[26]. MusalwaMuyangwal, EkaterinaV.Martynoval, SvetlanaF.Khaiboullinal,2 SergeyP.Morzunov3 and AlbertA.Rizvanov1 Hanta viral Proteins: Structure, Functions, and Role in Hantavirus Infection FrontiersinMicrobiology|www.frontiersin.org 7 November2015|Volume6|Article1326

[27]. Satoshi Fukuyama1 and Yoshihiro Kawaoka1,2,3,4 The pathogenesis of influenza virus infections: the contributions of virus and host factors Curr Opin Immunol. 2011 August ; 23(4): 481-486.

[28]. Bernadeta Dadonaite 1,5, Brad Gilbertson 2,5, Michael L. Knight 1, Sanja Trifkovic 2,Steven Rockman2,3, Alain Laederach 4, Lorena E. Brown 2*, Ervin Fodor $1^{*}$ and David L. V. Bauer $1^{*}$ The structure of the influenza A virus genome Nature Microbiology | VOL 4 | NOVEMBER 2019 | 17811789

[29]. Author manuscript; available in PMC 2008 August 11. Jeffery K. Taubenberger and David M. Morens The Pathology of Influenza Virus Infections Annu Rev Pathol. 2008 ; 3: 499-522.

[30]. Shamimual Hasan et al , Dengue virus: A global human threat: Review of literature J Int Soc Prev Community Dent Jan-feb 2016; 6(1):1-6

[31]. Hindawi Publishing CorporationJournal of Immunology Research Volume 2016, Article ID 6803098, 14 pages Review Article Dengue Fever: Causes, Complications, and Vaccine Strategies Niyati Khetarpal1,2 and Ira Khanna1

[32]. M A Rosid*, A S Fitrani, Y Findawati, S Winata, and V A Firmansyah Classification Of Dengue Hemorrhagic Disease Using Decision .Tree With Id3 Algorithm Journal of Physics: Conference Series 1381 (2019) 012039

[33]. Ganeshkumar P, Murhekar MV, Poornima V, Saravanakumar V, Sukumaran K, Anandaselvasankar A, et al. (2018) Dengue infection in India: A systematic review and meta-analysis. PLoS Negl Trop Dis 12(7):2-29 e0006618 PLOS Neglected Tropical Diseases
[34]. Vaddadi Srinivas, Vaddadi Radha Srinivas. "Dengue Fever: A Review Article". Journal of Evolution of Medical and Dental Sciences 2015; Vol. 4, Issue 29, April 09; Page: 5048-5058,

[35]. Courtney P. Long1,2, Sarah M. McDonal PLoS Pathog 13(4) April20,2017 PEARLS Rotavirus genome replication: Some assembly required

[36]. Robert F. RamigRobert F. Ramig* MINIREVIEW Pathogenesis of Intestinal and Systemic Rotavirus Infection JOURNAL OF VIROLOGY, Oct. 2004, p. 10213-10220 VOL. 78, No. 192004

[37]. Harry B. Greenberg, M.D. Mary K. Estes, Ph.D. Rotaviruses: from pathogenesis to vaccination Gastroenterology. 2009 May ; 136(6): 1939-1951

[38]. Jiang Gu and Christine Korteweg Review Pathology and Pathogenesis of Severe Acute Respiratory Syndrome The American Journal of Pathology, Vol. 170, No. 4, April 2007

[39]. SARS coronavirus infection: pathology and pathogenesis of an emerging virus disease Sherif R. Zaki and Cynthia S. Goldsmith Infectious Disease Pathology Activity, Division of Viral and Rickettsial Diseases, NationalCenter for Infectious Diseases, Centers for Disease Control and Prevention (CDC), Atlanta, GA 30333, USA Coronaviruses with Special Emphasis on First Insights Concerning SARS 87 ed. by A. Schmidt, M.H. Wolff and O. Weber

[40]. I-Mei Yuł1,Michael L. Oldham $\$ 1$, Jingqiang Zhang§, and Jue Chent Crystal Structure of the Severe Acute Respiratory Syndrome(SARS) Coronavirus Nucleocapsid Protein Dimerization Domain Reveals Evolutionary Linkage between Corona- and ArteriviridaeI- THE JOURNAL OF BIOLOGICAL CHEMISTRY VOL. 281, NO. 25, pp. 17134-17139, June 23, 2006

[41]. Structure, Function, and Evolution of Coronavirus Spike Proteins Annu Rev Virol. 2016 September 29; 3(1): 237-261. doi:10.1146/annurev-virology-110615042301 doi:10.20944/prepr

[42]. Critical Differences between the Binding Features of the Spike Proteins of SARS-CoV-2 and SARS-CoV Chen Bai and Arieh Warshel* Cite This: J. Phys. Chem. B 2020, 124, 5907-5912

[43]. COVID-19 Pandemic: Insights into Structure, Function, and hACE2 Receptor Recognition by the SARS-CoV-2 Anshumali Mittal1*, Kavyashree Manjunath2, Rajesh Kumar Ranjan3, Sandeep Kaushik4, Sujeet Kumar5, Vikash Verma6*Preprints (www.preprints.org) | NOT PEER-REVIEWED | Posted: 15 May 2020

[44]. M.artina Bianchi ,1 Domenico Benvenuto ,2 Marta Giovanetti ,3 Silvia Angeletti ,4 Research Article Sars-CoV-2 Envelope and Membrane Proteins: Structural Differences Linked to Virus Characteristics? BioMed Research International Volume 2020, Article ID 4389089, 6 pages https://doi.org/10.1155/2020/4389089 